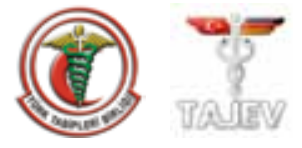

\title{
Multiple sclerosis; a disease of reproductive-aged women and the dilemma involving contraceptive methods
}

\author{
Esengül Türkyılmaz ${ }^{1}$, Melahat Yıldırım ${ }^{1}$, Ayşe Filiz Yavuz Avşar ${ }^{2}$ \\ 'Department of Obstetrics and Gynecology, Atatürk Training and Research Hospital, Bilkent, Ankara, Turkey \\ ${ }^{2}$ Department of Obstetrics and Gynecology, Yildırm Beyazıt University, Bilkent, Ankara, Turkey
}

\begin{abstract}
Multiple sclerosis (MS) is an autoimmune disorder characterized by chronic inflammation in the central nerves system. Because the disease predominantly affects women of reproductive ages, having knowledge about contraception options for MS patients can make clinicians provide better counseling. Although most contraceptive methods are generally accepted as safe and effective in MS patients, recent studies have raised questions about their potential adverse effects on the disease. The use of contraceptive methods to avoid unintended pregnancies is crucial in MS patients, particularly during the relapse phase of the disease or the time when the disease is not completely under control. This review investigates the contraception options and their effects on female MS patients.

Providing appropriate contraception options to multiple sclerosis patients will be one of the most challenging issues for clinicians to deal with. Recent studies have raised questions that the use of hormonal contraceptives may at least partly contribute to the rise in incidence of MS in women. This review investigates the contraception options and their effects on female MS patients.
\end{abstract}

(J Turk Ger Gynecol Assoc 2015; 16: 49-53)

Keywords: Multiple Sclerosis, contraception, contraceptives

Received: 01 October, 2014

Accepted: 08 January, 2015

\section{Introduction}

Multiple sclerosis (MS) is an autoimmune disorder characterized by chronic inflammation in the central nerves system and subsequent demyelination of nerves (1).The disease predominantly affects women of reproductive ages in the $3^{\text {rd }}$ and $4^{\text {th }}$ decades; $62 \%$ of these women use contraceptive methods $(2,3)$. There are recent conflicting studies about the effect of oral contraceptive (OC) use on MS and MS-like symptoms in the literature $(4,5)$. Therefore, it is important to understand the effects of hormonal contraceptives in MS patients.

In addition, the medical treatments of MS such as through the use of interferons (IFNs), fingolimod, dimethyl fumarate, and, natalizumab may compromise the pregnancy in patients with this disease (6). The ongoing use of IFNs in women planning pregnancy may also be relatively contraindicated because of possible abortifacient properties (6). Therefore, the use of contraceptive methods to avoid unintended pregnancies will be extremely important in MS patients, particularly during the relapse phase of the disease or the time when the disease is not completely under control.
Therefore, clinicians usually try to provide contraceptive counseling to these young MS patients for addressing the question regarding the most appropriate contraceptive method. Providing an appropriate contraception options to MS patients is one of the most challenging issues for clinicians to deal with. However, most contraceptives methods are hormonal methods, and recent studies have raised questions about their potential adverse effects on the disease.

We start this review by discussing the use of hormonal contraceptives in MS patients. To understand the effect of hormonal contraceptives on MS, the effects of sex steroids on this disease are discussed initially.

\section{MS, sex steroids, hormonal contraceptive methods}

Although the strong interaction between the pathogenesis of MS and the immune system and sex steroids is well known for a long time, the exact mechanism through which the disease develops is yet to be identified completely. Hormonal contraceptives are effectively used by many young women to prevent unintended pregnancies.

Most women use this method, particularly in their middle reproductive years, in which MS is mostly detected. The well-recognized problem in the pathogenesis of MS is inflam- 
mation of the myelin and the defects in the regeneration of the myelin sheaths. The defective regeneration of myelin sheaths as a result of inflammation shows up as a white region causing plaque appearance in the nerve system (7).

Observations such as the alleviation of the symptoms of MS during pregnancy when the progesterone level becomes high, the increase in the frequency of attacks after the delivery when progesterone levels rapidly decrease (7), the aggravation of the symptoms of the disease during the premenstrual period (8), and the increase in relapses following the end of progesterone treatment during the assisted reproductive treatment (9) suggest that progesterones can provide a mitigating effect against the development of MS attacks. Based on the aforementioned observations, it was recommended that nomegestrol acetate (NOMAC), a highly selective progestogen with the ability to cross the blood-brain barrier easily, may be used to help prevent the relapse of the disease.

NOMAC, derived from 19-nor-progesterone, suppresses the secretion of gonadotropin-releasing hormone (GnRH) from the hypothalamus and inhibits the production of progesterone from ovaries (10). It was shown in animal studies that NOMAC increases the production of ALLOPREG, which plays an important role in the coordination between neurons and supporting cells, which are required for producing myelin sheaths (11).

It is well documented that the mechanism of action of progestin only pills (POPs) is basically related to their progestogenic effect of thickening the cervical mucus, and thinning of the endometrium causes it to become involuted. Thus suppressant effect of progesterones on the endometrium prevents the implantation of the product of conception (12). POP is usually preferred during the postpartum period for its minimally suppressive effect on lactation. Considering the effect of progesterone on the myelin sheaths, it can be argued that POP can be an effective choice of contraception for MS patients during the postpartum period.

Based on this discussion, it can be expected that other progesterone preparates such as medroxyprogesterone acetate injection (Provera, Depo-Provera) and the progestin preparates etonogestrel subdermal implant (Implanon) or levonorgestrel implants (Norplant and Jadelle) will have a similar progestogenic effect on the neuronal system in MS patients. However, rising rumors about the presence of MS-like symptoms in women with levonorgestrel-releasing intrauterine devices (LNG-IUDs) increasingly cast doubts on the use of progestins as contraceptive methods (13).

The effect of progestin in LNG-IUD is mainly local, e.g., causing glandular atrophy and decidualization of the endometrium, resulting in thickening of the cervical mucus, and creating a barrier to sperm penetration (12). The contraceptive effect of LNG-IUD basically depends on its spermicidal effect and partially depends on the inhibition of follicle maturation and the inhibition of ovulation (12). At present, two LNG-IUDs, Mirena ${ }^{\circledR}$ (Schering Health, Berlin, Germany) and Skyla (Bayer, Pittsburgh, Pennsylvania) are on the market. In this review, LNG-IUD refers to the Mirena ${ }^{\circledR}$ IUD because little clinical research currently exists regarding the Skyla ${ }^{\circledR}$ IUD. Levonorgestrel in Mirena ${ }^{\circledR}$ is released into the blood circulation minimally, approximately
20 mcg per day, and its amount tends to decrease with THE duration of use (12). In addition to the supposed protective the effect of progestins against demyelination in MS patients, the negligible amount of levonorgestrel found in the bloodstream of LNG-IUD users does not support the argument that LNG-IUD causes MS or MS-like symptoms in women. However, in recent years, a growing number of cases regarding the development of MS symptoms in women using Mirena ${ }^{\circledR}$ have prompted significant concern by both MS patients and clinicians (13). In a recent study, 62,517 female participants were directed to fill out web-based questionnaires regarding the use of Mirena ${ }^{\circledR}$ and MS diagnosis. It was reported that out of 62,517 registered women with Mirena ${ }^{\circledR}, 31$ were diagnosed with MS. In total, $20 \%$ of these women were diagnosed with the disease within a month of IUD insertion, while 30\% developed MS between 1-6 months, 10\% between 2-5 years, and 10\% between 5-10 years. While the incidence of MS among women without Mirena ${ }^{\circledR}$ was $0.01 \%$, this rate increased to $0.05 \%$ among women with Mirena ${ }^{\circledR}$ (13). The overall incidence rate of MS in women was found to be $0.036 \%$ in another study (14). However, it is hard to interpret the aforementioned results because of the lack of prospective controlled studies and the limited data regarding women who may have early MS symptoms that are vague and undiagnosed at the time of the insertion of LNG-IUD. Nevertheless, one should always take into account the possibility of exacerbation of the disease in patients diagnosed with MS while recommending Mirena ${ }^{\circledR}$ or possibly Skyla.

There are a number of experimental studies regarding the effect of estrogens on the development and progression of MS. In one of the most typical of these studies, experimental autoimmune encephalomyelitis was induced in mice, and estradiol and ethynil estradiol were administered to investigate the effect of estrogens on neurons (15). This and other related studies have shown that estrogens decrease the production of tumor necrosis factor- $\alpha$ (TNF $\alpha)$ and other chemokines $(15,16)$, inhibit major histocompatibility complex-II (MHC-II) expression (17), and protect dendritic cells from inflammation (18). After the administration of estrogens in the study, the decrease in the production of TNF$\alpha$, interferon gamma (IFN-gamma), and interleukin-12 (IL-12) in dendritic cells; the decrease in antigen presentation to $\mathrm{T}$ cells (19); and a shift from T helper-1 (TH-1) to T helper-2 (TH-2) cells were observed (19). Moreover, both estrogen- and progesteronecontaining OCs have been shown to promote the survival and growth of neurons and myelin formation (20). In addition, it is reported that the alleviation of symptoms of MS during pregnancy is related to enhanced immune response arising from high estrogen levels (21). Based on these studies, it was concluded that estrogens can prevent patients from developing MS symptoms by affecting the immune response and inflammation. Based on these findings, it was suggested that estrogen-containing agents can be the choice of both contraceptive methods and treatment for MS in young women with this disorder. However, this hypothesis is not supported by clinical studies. Moreover, the question of why more women have MS than men if estrogen prevents the disease has remained unanswered.

There are four large prospective studies performed regarding OCs and their effects on MS. One of those studies, the Oxford 
Family Planning Association Study (4) was conducted between 1968-1974 on a total of 17,032 female patients aged between 25-39 years who presented to family planning clinics requesting contraceptive counseling. The main aim was to investigate the efficacy of contraceptive methods and their effects on the general health. In total, $56 \%$ of the women were using OCs for birth control. No correlation between the use of OC and MS was detected in the study (4). Another study named the Royal College of General Practitioners' Oral Contraception Study (22) investigated 46,000 women, half of whom used OCs as contraceptive methods in 14 months of the study period. The previous OC users, new OC users, and nonusers were compared in terms of the risk of developing MS, and no correlation was found between these variables (22). Furthermore, no significant association was observed between the risk of MS and OC medicines that contain a different combination of active ingredients ( $<50 \mu \mathrm{g}$ estrogen, $>50 \mu \mathrm{g}$ estrogen, progesterone only) in the study.

In the Nurses' Health Study (NHS), 121,700 OC user patients aged between 30-55 years were followed up for at least 6-7 years. The Nurses' Health Study II (NHSII) included 116,671 women aged between 25-42 years who used OCs as contraceptive methods (23). In total, 181 women in NHS and 134 women in NHSII developed MS during the 6-7-year follow-up. Although the increase in the risk of the development of MS was found in OC users, no correlation between the duration of $\mathrm{OC}$ use and the risk of MS was detected (23).

In the study of General Practice Research Database (24), the study group consisted of 106 women with MS and the control group consisted of 1001 healthy women. They found that women who previously used OCs and had just stopped it had a decreased risk of developing MS in comparison with nonusers (24). They showed that the incidence of MS was $40 \%$ lower in OC users than in nonusers during the previous 3 years. The risk of MS increased in the 6 months after pregnancy. However, conclusions derived from the short-term study were not valid in the long term (24). None of these studies were convincing enough to reveal that the use of OCs increases the incidence of MS. Based on these studies, it was previously concluded that hormonal contraceptives were used as contraceptive options for patients with MS or MS-like symptoms. However, the findings of the study presented in the 66th Annual Meeting of American Academy of Neurology in 2014 have changed the general concept about the effects of hormonal contraceptives on MS patients (5). In this population-based nested case-control study, 305 incident female cases with MS and 3050 matched controls were identified. It was observed that $29.2 \%$ of women with MS and $23.5 \%$ of control patients had used hormonal contraceptives for at least 3 months within the 3 years prior to the onset of symptoms. The majority of women used estrogen/progestin combination preparations. Women who used any hormonal contraceptive in the 3 years prior to the onset of symptoms, particularly those who had stopped the use at least 1 month prior to the beginning of symptoms, had a slightly increased risk of MS (5). Based on their findings, they suggested that the use of hormonal contraceptives at least partly contributes to the rise in the incidence of MS in women (5).
It seems that the opinion about the use of hormonal contraceptives in MS patients tends to change after this study. These contraceptive methods may not be as innocent as they seem in MS patients. Therefore, clinicians should be aware of the potential harmful effect of hormonal contraceptives in patients with known or suspected MS. If they have undiagnosed MS symptoms, they may be more likely to avoid pregnancy and to use hormonal contraceptives. If they have vague symptoms while on a pill prior to the diagnosis of MS, they may stop the pill to see whether their symptoms are related to the use of hormonal contraceptives.

It is also expected that certain drugs used to treat MS may reduce the effectiveness of OCs in these patients. It should be kept in mind that there is an increased risk of thrombosis associated with immobility in MS patients. In the presence of these conditions, other alternative contraceptive options should be recommended to MS patients.

The observations that MS is rare among men in general suggest the possible protective effects of androgens against the disease (25). The recent experimental study has revealed that testosterone treatment efficiently stimulates the formation of new myelin and reverses myelin damage in chronic demyelinated brain lesions in organotypic culture (25). In addition to the strong effect of testosterone on myelin repair, the authors of that study observed that the number of activated astrocytes and microglial cells returned to low control levels, indicating a reduction of neuroinflammatory responses. They have also identified the neural androgen receptor as a novel therapeutic target for myelin recovery (25). They concluded that androgens can be used as remyelinating agents and the brain androgen receptors can be promising drug targets for remyelination therapy in male MS patients (25).

It is well known that some OCs, LNG-IUDs, and LNG implants contain progesterone derivatives, which have more potent androgenic effects than the others. This feature may prove helpful in deciding the type of OCs in women with MS. Needless to say, to demonstrate the effect of the androgenic progestins on MS, future prospective studies should be performed.

In conclusion, given the lack of clarity regarding the use of hormonal contraceptives in MS patients and their potential risk of adversely affecting MS or MS-like symptoms in female patients, contraceptive methods should be carefully selected after considering the balance between their advantage and disadvantage.

\section{MS and nonhormonal contraception}

Inflammation plays an important role in the pathogenesis of MS. Vascular endothelial growth factor (VEGF), an angiogenic growth factor, promotes the resistance against neuronal damage and regulates the proliferation of neuroprogenitor cells, migration, differentiation, and survival of oligodentrocyte precursor cells, and migration to demyelinated lesions (26). However, in chronic MS, angiogenesis induced by inflammation seems to be limited because of a counterbalancing effect of vasoconstrictive mechanisms (26). During the chronic phase of MS, responsiveness to VEGF lessens and the regenerative process becomes disrupted (26). Angiogenesis in MS brings about 
neovascularization and causes a rise in the vascular supply of nutrients and migration of inflammatory cells to demyelinating lesions (27). Copper-containing IUDs are among the most commonly used contraceptive options. The mechanism of action of the copper IUD is based on the direct effect on the oocytes via lessening or inhibiting their fertilization capacities $(28,29)$. This device has also an effect on the uterine cavity, endometrium, and cervical mucus, resulting in the immobilization of sperms and prevention of sperms to migrate to the fallopian tubes (28). Copper-containing IUDs release free copper and copper salts into the uterine cavity without increasing serum copper levels in women with IUDs (30). Early analyses suggested that IUDs cause pelvic inflammatory disease (PID) in women. However, more recent studies have found no increased risk of PID in monogamous women with IUDs (31-33). It can be assumed that MS attacks can be triggered by the presence of infection in the genital tract. Based on the data in literature, it can be concluded that copper-containing IUDs can be safely used by MS patients. However, this hypothesis should be supported by prospective controlled studies. It is well known that the use of diaphragms increases urinary tract infections in women (34). The presence of infections can induce MS symptoms with mechanisms similar to those mentioned above.

\section{Conclusion}

Because MS is usually observed among women of the reproductive age, the question about the most reliable contraceptive option for these patients will always be the first one for clinicians to answer. While the earlier research did not oppose the idea of using hormonal contraceptives in MS patients, a recent study has suggested that certain hormonal methods may increase the risk of developing MS or MS-like symptoms. Based on recent findings, we recommend that clinicians should prescribe selective nonhormonal contraceptives such as copper-containing IUDs in all phases of MS disease to be on the safe side. In addition, when counseling about contraception methods for MS patients, each case should be individually evaluated on the basis of the severity of the disease and patient's lifestyle instead of steering away from this issue on the basis of inconclusive data. Further studies are required to arrive at a definitive conclusion regarding the identification of potential adverse effects of various hormonal and nonhormonal contraceptive methods.

\section{Peer-review: Externally peer-reviewed}

Author contributions: Concept - M.Y., E.T.; Design-E.T., M.Y.; Supervision A.F.Y.A.; Resource - M.Y.; Materials - E.T.; Data Collection\&/or Processing - M.Y., E.T.; Analysis\&/or Interpretation - E.T., M.Y.; Literature Search M.Y., E.T.; Writing - E.T., M.Y.; Critical Reviews - A.F.Y.A.

Conflict of Interest: No conflict of interest was declared by the authors.

Financial Disclosure: The authors declared that this study has received no financial support.

\section{References}

1. Noseworthy JH, Lucchinetti C, Rodriguez M, Weinshenker BG. Multiple sclerosis. N Engl J Med 2000; 343: 938-52. [CrossRef]
2. Jones J, Mosher WD and Daniels K. Current contraceptive use in the United States, 2006-2010, and changes in patterns of use since 1995, Natl Health Stat Report 2012; 18: 1-25.

3. http://www.guttmacher.org/media/presskits/contraception-US/ index.html, Contraceptive Use in the United States, March 2014.

4. Vessey MP, Lawless M. The Oxford- Family Planning Association contraceptive study. Clin Obstet Gynaecol 1984; 11: 743-57.

5. Rachel Seroka, Michelle Uher. Hormonal contraceptives and Multiple Sclerosis Susceptibility. AAN 66 th Annual Meeting Abstract. 2014-08-21.

6. Available online: http://www.clevelandclinicmeded.com/medicalpubs/diseasemanagement/neurology/multiple_sclerosis/ Robert J. Fox. Multiple Sclerosis, June 2014.

7. Helmut Leitner. Multiple sclerosis and progestins: A comment POPART'MUS. J Neurol Sci 2011; 300: 198-9. [CrossRef]

8. Wilson S. Premenstrual worsening of MS symptoms. Way Ahead 2001; 5: 14.

9. Hellwig K, Schimrigk S, Beste C, Muller T, Gold R. Increase in relapse rate during assisted reproductive technique in patients with multiple sclerosis. Eur Neurol 2009; 61: 65-8. [CrossRef]

10. Couzinet B, Young J, Kujas M, Meduri G, Brailly S, Thomas JL, et al. The antigonadotropic activity of a 19-nor-progesterone derivative is exerted both at the hypothalamic and pituitary levels in women. J Clin Endocrinol Metab 1999; 84: 4191-6. [CrossRef]

11. Lenzi E, PluchinoN, Begliuomini S, Ninni F, Freschi L, Centofanti M, et al. Effects of nomegestrolacetat administration on central and peripheral beta-endorphin and allopregnanolone in rats. J Steroid Biochem Mol Biol 2008; 110: 67-75. [CrossRef]

12. Leon Sperof, Marc A. Fritz. Klinik Jinekolojik endokrinoloji ve infertilite. Sekizincibaskı.2014; Bölüm 25. p.1102

13. Available online: Review: could Mirena cause Multiple Sclerosis. November 2014, http://www.ehealthme.com/ds/mirena/multiple+ sclerosis.

14. Alanso A, Hernan MA. Temporal trends in the incidence of multiple sclerosis, A systematic review. Neurology 2008: 71; 129-35. [CrossRef]

15. Ito A, BeboJr BF, Matejuk A, Zamora A, Silverman M, Fyfe-Johnson A, Offner $\mathrm{H}$. Estrogen treatment down-regulates TNF-alpha production and reduces the severity of experimental autoimmune encephalomyelitis cytokine knockout mice. J Immunol 2001; 167: 542-52. [CrossRef]

16. Matejuk A, Adlard K, Zamora A, Silverman M, Vandenbark AA, Offner $\mathrm{H}$. 17 beta-estradiol inhibit cytokine, chemokine receptor mRNA expression in the central nervous system of female mice with experimental autoimmune encephalomyelitis. J Neurosci Res 2001; 65: 529-42. [CrossRef]

17. Adamski J, Ma Z, NozellS, Benveniste EN. 17 beta-estradiol inhibit class II major histocompatibility complex (MHC) expression: influence on histone modifications and CBP recruitment to the class II MHC promoter. Mol Endocrinol 2004; 18: 1963-74. [CrossRef]

18. Liu HY, Buenafe AC, Matejuk A, Ito A, Zamora A, Dwyer J, et al. Estrogen inhibition of EAE involves effects on dendritic cell function. J Neurosci Res 2002; 70: 238-48. [CrossRef]

19. Egemen İdiman. Multipl skleroz ve gebelik. Archives of Neuropsychiatry 2008; 45: 21-5.

20. El-Etr M, Ghoumari A, Sitruk-Ware R, Schumacher M. Hormonal influences in multiple sclerosis: new therapeutic benefits for steroids. Maturitas 2011; 68: 47-51. [CrossRef]

21. El-Etr M, Vukusic S, Gignoux L, Durand-Dubief F, Achiti I, Baulieu EE, Confavreux C. Steroid hormones in multiple sclerosis. J Neurol Sci 2005; 15: 233: 49-54. [CrossRef]

22. Hannaford PC, Selveraj S, Elliott AM, Angus V, Iversen L, Lee AJ. Cancer risk among users of oral contraceptives: cohort data from 
the Royal College of General Practitioner's oral contraceptives: cohort data from the Royal College of General Practitioner's oral contraception study. BMJ 2007; 335: 651. [CrossRef]

23. Hernàn MA, Hohol MJ, Olek MJ, Spiegelman D, Ascherio A. Oral contraceptives and the incidence of multiple sclerosis. Neurology 2000; 55: 848-54. [CrossRef]

24. Alonso A, Jick SS, Olek MJ, Ascherio A, Jick H, Hernán MA. Recent use of oral contraceptives and the risk of multiple sclerosis. Arch Neurol 2005; 62: 1362-5. [CrossRef]

25. Hussain R, Ghoumari AM, Bielecki B, Steibel J, Boehm N, Liere $P$, et al. The neural androgen receptor: a therapeutic target form myelin repair in chronic demyelination. Brain 2013; 136: 132-46. [CrossRef]

26. Girolamo F, Coppola C, Ribatti D, Trojano M. Angiogenesis in multiplesclerosisandexperimentalautoimmuneencephalomyelitis. Acta Neuropathol Commun 2014; 1: 84. [CrossRef]

27. Griffioen AW, Molema G. Angiogenesis: potentials for pharmacologic intervention in the treatment of cancer, cardiovascular diseases, and chronic inflammation. Pharmacol Rev 2000; 52: 237-68.

28. Ortiz ME, Croxatto HB: The mode of action of IUDs. Contraception 1987; 36: 37-53. [CrossRef]
29. Wilcox AJ, Weinberg CR, Armstrong EG, Canfield RE. Urinary human chorionic gonadotropin among intrauterine device users: Detection with a highly specific and sensitive assay. Fertil Steril 1987; 47: 265-9.

30. Newton J, Tacchi D: Long-term use of copper intrauterine devices. A statement from the Medical Advisory Committee of the Family Planning Association and the National Association of Family Planning Doctors. Lancet 1990; 335: 1322-3. [CrossRef]

31. Grimes DA. The intrauterine device, pelvic inflammatory disease, and infertility: The confusion between hypothesis and knowledge. Fertil Steril 1992; 58: 670-3.

32. Kessel E. Pelvic inflammatory disease with intrauterine device use: A reassessment. Fertil Steril 1989; 51: 1-11.

33. Lee NC, Rubin GL, Borucki R. The intrauterine device and pelvic inflammatory disease revisited: New results from the Women's Health Study. Obstet Gynecol 1988; 72: 1-6.

34. Fritz MA, Speroff L. Clinical Gynecologic Endocrinology and Infertility. Wolter Kluwer/Lippincott Williams \&Wilkins. 2014; 26: 1125 . 